\title{
PRAGMÁTICA E IRONÍA VERBAL: REVISIÓN CRÍTICA Y CLAVES TEÓRICO-METODOLÓGICAS
}

\author{
PRAGMATICS AND VERBAL IRONY: CRITICAL REVIEW AND \\ THEORETICAL-METHODOLOGICAL KEYS
}

\author{
LaURa de la CASA Gómez \\ Universidad de Jaén, España \\ Grupo de investigación Análisis lingüístico: \\ Teoría y aplicaciones (ALTYA) (HUM-834) \\ lcgomez@ujaen.es \\ https://orcid.org/0000-0002-9616-1831
}

\begin{abstract}
Resumen
La compleja naturaleza de la ironía verbal ha llevado a los lingüistas a tratar de definirla a partir de perspectivas de análisis tan heterogéneas que la amplia bibliografía al respecto genera más dilemas de los que resuelve, ya que ninguna es por sí sola suficiente para caracterizar nítidamente la totalidad de manifestaciones irónicas posibles. A la luz de este problema teórico, en este trabajo presentamos una revisión y análisis de cinco de los modelos teóricos que, dentro de la disciplina pragmática, han tenido mayor trascendencia en el estudio de la ironía verbal. Nuestros objetivos son mostrar sus debilidades teóricas, así como los fundamentos que son potencialmente válidos, exponiendo las razones al respecto, para, a partir de las conclusiones
\end{abstract}

\begin{abstract}
Verbal irony is so complex in nature that linguists have tried to define it from perspectives of analysis very heterogeneous. Consequently, an extensive bibliography on the matter is available. However, it generates more dilemmas than it solves, since no theory by itself is sufficient to typify the totality of possible ironic occurrences clearly. Given this theoretical problem, this work presents a review and analysis of five of the most significant theoretical models in the study of verbal irony within the pragmatics discipline. The aim is, in the first place, to show their theoretical weaknesses, as well as the foundations that are potentially valid, providing reasons in that regard; and, in second place, to expound some of the theoretical
\end{abstract}

Para citar este artículo: De la Casa Gómez, Laura (2021). Pragmática e ironía verbal: revisión crítica y claves teórico-metodológicas. ELUA, 35: 73-92. https://doi.org/10.14198/ELUA2021.35.4

Recibido: 11/05/2020, Aceptado: 22/10/2020

(C) 2021 Laura de la Casa Gómez

Este trabajo está sujeto a una licencia de Reconocimiento 4.0 Internacional

de Creative Commons (CC BY 4.0) 
extraídas, ofrecer algunas de las claves teóricas y metodológicas esenciales para lograr avanzar hacia una definición más unitaria, abarcadora y consistente de la ironía verbal.

PALABRAS CLAVE: ironía verbal; definición; revisión crítica; pragmática. and methodological keys that the conclusions drawn previously suggest as essential to progress towards a more unitary, comprehensive and consistent definition of verbal irony.

KEYWORDS: verbal irony; definition; critical review; pragmatics.

\section{INTRODUCCIÓN}

Numerosos investigadores (Gibbs y O’Brien 1991; Littman y Mey 1991; Alba Juez 1995; Torres Sánchez 1999; Rodríguez Rosique 2009; Ruiz Gurillo 2010) han puesto de relieve la dificultad —y casi imposibilidad — de proponer una definición nítida y absoluta de la ironía verbal. Este problema teórico viene causado por la compleja naturaleza del fenómeno, ya que, por un lado, es muy amplia, dada la gran variedad de factores que intervienen en su producción e interpretación, y, a la vez, es muy singular, debido a que su sentido depende de cada contexto particular de uso (Marimón 2009: 40; Ruiz Gurillo 2010: 96). En consecuencia, disponemos de una extensa bibliografía dedicada al estudio de la ironía verbal que, sin embargo, más que contribuir a la tarea de elaborar una definición unitaria, la obstaculiza, pues los enfoques adoptados son tan heterogéneos ${ }^{1}$ que solo alcanzan a explicar, en palabras de Colston y Gibbs (2007: 4), "a portion of the phenomenon, or one of a variety of mechanisms underlying [its] comprehension".

En este trabajo presentamos una revisión del concepto de ironía verbal a partir del análisis de cinco de los modelos teóricos que han tratado de explicarla dentro de la disciplina pragmática, a saber, el análisis pragmalingüístico de Haverkate (1985), la teoría de la mención de Sperber y Wilson $(1981,1986)$, la teoría de la manifestación implícita de Utsumi (2000), la teoría de la inadecuación relevante de Attardo (2000) y el modelo neogriceano del grupo GRIALE (Ruiz Gurillo y Padilla, eds. 2009)². A continuación, aplicamos los fundamentos de dichas teorías en ejemplos irónicos pertenecientes a un corpus oral de elaboración propia, a fin de comprobar o refutar las conclusiones extraídas tras la revisión crítica anterior. Nuestros objetivos son mostrar las deficiencias teóricas de estos modelos, así como los fundamentos potencialmente válidos, exponiendo las razones al respecto, para finalmente ofrecer algunas de las claves teóricas y metodológicas esenciales para lograr avanzar hacia una definición más abarcadora y coherente de la ironía verbal.

Nuestra investigación parte de un estudio de corpus compuesto por más de cuatrocientos ejemplos de ironías en español, extraídos de tres programas televisivos españoles:

1 Los presupuestos de las diversas perspectivas de análisis provienen de disciplinas con trasfondos muy variados, principalmente, filosóficos, retóricos, lingüísticos y psicológicos.

2 Cabe citar otras aproximaciones teóricas de gran relevancia en la caracterización de la ironía verbal, como la teoría de Grice $(1975,1978)$ — que pertenece al enfoque pragmático tradicional—, la teoría polifónica de Ducrot (1984) — dentro de los estudios sobre el discurso — , las teorías del fingimiento de Clark y Gerrig (1984) y de la alusión insincera a las expectativas rotas del hablante de Kumon-Nakamura et alii (1995) —ambas de corte psicolingüístico-, y la teoría de la negación indirecta de Giora $(1995,1997)$ — que en parte sigue un enfoque neogriceano-. No obstante, puesto que los fundamentos de todas ellas son posteriormente reelaborados para constituir las bases de los modelos que aquí analizamos — que, además, tienen mayor vigencia—, no las incluimos en la revisión. 
La Sexta Noche, Sábado Deluxe y First Dates ${ }^{3}$. Los enunciados irónicos se emplean en interacciones de naturaleza discursiva diferente: el debate periodístico, la entrevista y la conversación coloquial.

\section{EL ESTUDIO PRAGMÁTICO DE LA IRONÍA VERBAL}

\subsection{La ironía como un acto de habla insincero}

Haverkate (1985), en su análisis pragmalingüístico, concibe la ironía como una insinceridad transparente del hablante que puede encontrarse en dos niveles del enunciado, el proposicional y el ilocutivo. El primer caso ocurre cuando el hablante rompe con la condición de sinceridad de un acto de habla asertivo, lo que genera una implicatura que deriva en un efecto de contrariedad o negación del contenido proposicional. Por ejemplo, un enunciado como Me encanta esta armónica música expresado por un hablante que escucha a un desafinado violinista se interpretaría como lo contrario de lo aseverado, que le resulta desagradable escuchar un sonido tan disonante. El segundo caso de ironía tiene lugar cuando el hablante rompe una o varias condiciones de adecuación de cualquiera de los otros tipos de actos de habla, lo que tiene como posibles efectos la negación de su fuerza ilocutiva o el surgimiento de otra distinta, manifestándose como un acto de habla indirecto. Imaginemos a un padre que, tras ver a su hijo lastimarse manejando un martillo, le dice Muy bien, tú sigue haciéndote daño. La intención real del hablante no solo es negar el acto de habla directivo, la exhortación ('no continúes lastimándote'), sino también realizar un acto distinto, una prohibición ('suelta la herramienta').

Lo más destacable del análisis de Haverkate (1985) es que, además de ser sistemático, aplica presupuestos teóricos basados en la pragmática - concretamente, en la teoría de los actos de habla de Austin (1962) y Searle (1969)—, y no en la semántica — como ocurre en las explicaciones retóricas anteriores_-, de modo que ofrece una explicación del modo en que se interpreta el sentido irónico en función de la intención comunicativa que subyace a la expresión — que atañe a la actitud del hablante- . No obstante, el modelo es deficiente por una razón fundamental: la condición de la insinceridad pragmática no permite diferenciar la ironía de otros actos de habla indirectos, tales como las interrogaciones retóricas o las preguntas indirectas convencionales, en los que también puede afirmarse que las intenciones del hablante son ostensiblemente distintas de las expresadas, si bien no son irónicos. A este respecto Haverkate (1985) argumenta que, en una pregunta indirecta convencional no irónica, como ¿Podrías ayudarme a pintar mi casa?, el hablante sí desea realizar tanto el acto exhortativo implícito como el explícito primario, la petición de información. Sin embargo, en un enunciado como ¿Podrías hacerme el favor de callarte?, el hablante no es sincero con respecto al objetivo ilocutivo de la pregunta explícita, esto es, no espera una respuesta - sabe de antemano que su interlocutor es capaz de callarse - y, además, la cortesía expresada es fingida. Por ello, explica el autor, este ejemplo se interpreta irónicamente como algo distinto, en concreto, como la expresión de desprecio o enojo por parte del hablante.

A nuestro juicio, sus razonamientos son demasiado débiles e, incluso, infundados, ya que, en primer lugar, el acto implícito que llevan aparejado las preguntas indirectas no

3 Todos los programas fueron emitidos durante los meses de febrero y marzo de 2018. 
debe considerarse como un acto añadido al formalmente expresado, pues la razón de ser de un acto de habla indirecto, sea irónico o no, es la expresión de una fuerza ilocutiva - una intención - distinta de la que convencionalmente se asocia a la forma lingüística del enunciado, no adicional a ella. Y, en segundo lugar, la utilización deliberada de convenciones de cortesía en una situación comunicativa que hace evidente no solo su insinceridad, sino también "el carácter retórico" de la misma (Fernández García 2017: 360), para provocar, por ejemplo, un efecto descortés, es propia de otros mecanismos indirectos que no son irónicos, los enunciados ultracorteses, a los que, de hecho, consideramos que se adscribe el segundo ejemplo citado. Mientras que la insinceridad que manifiesta la ironía es pragmática —atañe a la fuerza ilocutiva del hablante, modificándola—, la de la ultracortesía es retórica — esto es, concierne a los efectos perlocutivos que el hablante desea generar-, por lo que, si cambiamos el perfil ultracortés del enunciado, su fuerza ilocutiva seguirá siendo la misma.

Para concluir con la revisión del análisis de Haverkate (1985), coincidimos con Barbe (1995: 52) en que, al estar arraigado en las teorías pragmáticas tradicionales, se centra en desentrañar las propiedades lógicas de la ironía a partir de enunciados aislados, sin tener en cuenta la incidencia del contexto comunicativo en el proceso interpretativo.

\subsection{La ironía como una mención ecoica}

De acuerdo con Sperber y Wilson $(1981,1986)$, la ironía verbal es una mención ecoica, ya que, a través de ella, el hablante se refiere de forma encubierta al contenido de un enunciado previo (eco explícito) o a una opinión o pensamiento atribuidos a personas específicas o grupos (eco implícito) con la finalidad de manifestar implícitamente su actitud despreciativa o de rechazo hacia dichos ecos. En cuanto al proceso de interpretación, la teoría de la mención es pionera en rechazar el modelo pragmático tradicional en dos etapas, que postula que el oyente debe percibir primero que el enunciado transgrede la máxima de cualidad del principio de cooperación de Grice (1975) para, posteriormente, recuperar el sentido irónico como una implicatura que contradice el significado literal. Por el contrario, Sperber y Wilson $(1981,1986)$ aplican en su modelo los fundamentos de la teoría de la relevancia y defienden que las inferencias que guían la interpretación irónica surgen porque el oyente reconoce que el enunciado es relevante en el contexto, dado que expresa la actitud del hablante hacia su contenido.

Los propios Sperber y Wilson (1981: 310) destacan que el aspecto teórico central de su propuesta, que la convierte en una alternativa teórica superior a las anteriores, es la consideración de la expresión de la actitud del hablante como el criterio caracterizador de la ironía verbal, ya que, en primer lugar, lleva a reflexionar sobre los motivos para el uso de este mecanismo implícito en la comunicación. En segundo lugar, la dimensión actitudinal de la ironía sirve para diferenciar esta de otros enunciados cuyo contenido literal también es abiertamente falso, pero no resultan irónicos — porque no manifiestan el estado anímico del hablante-; en tercer lugar, la atención a esta propiedad permite explicar una variedad más amplia de casos irónicos, pues la postura negativa del hablante puede deberse a que considera falso el contenido mencionado - como ocurre en la ironía estándar-, o a que le parece poco informativo, si bien no puede considerarse falso, como el enunciado Parece que llueve dicho justo en el momento en que arranca una fuerte tormenta. A propósito de estas ideas, reconocemos que la teoría de la mención pone en evidencia la necesidad de abordar otro 
tipo de enunciados irónicos no prototípicos — que no se corresponden con un significado literal falso- y avanza hacia la adopción de un nuevo enfoque que conciba la ironía como un mecanismo que, precisamente por su dimensión valorativa, sirve para la consecución de ciertas estrategias argumentativas, como presentar la perspectiva del interlocutor como absurda con el fin de desacreditarlo ${ }^{4}$.

No obstante, la propuesta cuenta con un gran número de detractores que, inicialmente, critican el hecho de que no alcanza a delimitar nítidamente la ironía de otro tipo de menciones ecoicas no irónicas, como la parodia. Wilson y Sperber (1992) admiten el fallo y proponen definir la ironía como una interpretación ecoica, ya que mediante ella el hablante metarrepresenta, no reproduce exactamente, un contenido que se asemeja a otra forma proposicional. A pesar de este cambio terminológico, el problema de la delimitación del fenómeno sigue sin solucionarse, porque, tal y como argumentan numerosos lingüistas (Clark y Gerrig 1984; Kumon-Nakamura et alii 1995; Giora 1995; Torres Sánchez 2009; Attardo 2000; Utsumi 2000), el concepto de eco es demasiado vago. Bruzos (2006: 35) opina que las condiciones que Sperber y Wilson $(1981,1986)$ establecen para definir el eco son prácticamente las mismas que las de cualquier otro tipo de discurso, y, además, Attardo (2000: 805) indica que son tan amplias que llevan a una "regresión infinita", dado que cualquier enunciado atribuido a un hablante hipotético es susceptible de considerarse como posible mención ecoica. Utsumi (2000: 1781) cuestiona la idea de que para reconocer la ironía sea necesario que el oyente relacione el contenido del enunciado irónico con el contenido de la fuente específica que se hace eco, pues señala que no es fácil vincular ambos contenidos debido a que, normalmente, no comparten implicaciones lógicas o contextuales, sino más bien relaciones de coherencia - $\mathrm{o}$, mejor dicho, de relevancia dentro del intercambio en curso- . Asimismo, estudiosos como Ruiz Gurillo (2010: 102) sostienen que la expresión de una actitud de disconformidad es una caracterización demasiado general y subjetiva, pues, como demuestra Giora (1995: 248), existen casos en los que un hablante se hace eco de las palabras de otro a la vez que expresa su actitud negativa hacia ellas, y, sin embargo, no son irónicos. Por tanto, tampoco es una condición suficiente para generar ironía, crítica que Wilson (2006: 1732) trata de justificar, sin éxito, apelando a la relatividad de sus presupuestos teóricos:

Within the range of interpretively used utterances, however, the borderline between $[\ldots]$ ironical and non-ironical attitudes [...] is much less clear-cut. In the first place, an utterance which is primarily intended as a report of speech or thought may be incidentally used to convey some information about the speaker's attitude [...]. In the second place, the prototypical ironical attitudes shade off into other types of dissociative or sceptical attitude, and a single utterance can convey a quite complex mixture of attitudes.

Ante las debilidades teóricas encontradas, Attardo (2000: 806) y Bruzos (2006: 38) proponen abandonar la caracterización de la ironía a partir de su naturaleza citativa y disociadora para centrar el interés en su condición de ser inadecuada en el contexto, pues ambos coinciden en afirmar que, si bien pueden darse casos de ironías cuyo contenido menciona otro (específico

4 Con base en su teoría de la enunciación polifónica, Ducrot (1984) analiza la ironía verbal centrándose especialmente en su naturaleza social e ideológica, determinando que la singularidad de la ironía radica en que posee un valor argumentativo. 
o posible), se trata de un hecho accidental e independiente del fenómeno ${ }^{5}$. Por nuestra parte, creemos que el planteamiento de estos autores de buscar en el factor contextual las claves para definir la ironía verbal, efectivamente, parece ser más acertado, dado que se trata de un fenómeno implícito que encierra cierta clase de contrariedad o incongruencia. Para concluir, estamos de acuerdo con Bruzos (2006: 54) en que el obstinado empeño de Sperber y Wilson por aportar una explicación de la ironía verbal basada en consideraciones experimentales de corte cognitivo les lleva a perderse en reflexiones teóricas, en su mayoría, insustanciales.

\subsection{La ironía como la manifestación implícita de un entorno irónico}

La teoría de la manifestación implícita de Utsumi (2000) trata fundamentalmente de dar respuesta a la cuestión de cómo se distingue un enunciado irónico de otro que no lo es. El autor opina que las teorías previas son incapaces de determinar qué elemento activa el proceso de reconocimiento del enunciado irónico, y, además, centran inútilmente sus esfuerzos en establecer las características identificables y comunes a todas las manifestaciones irónicas. Es por esto por lo que propone un prototipo de la ironía verbal, de manera que cuanto mayor sea el grado de semejanza entre un enunciado concreto y dicho modelo prototípico, más efectos irónicos generará y más fácil será para el oyente reconocerlo. Para exponer las bases de la teoría de la manifestación implícita, partamos de la situación presentada en el mismo estudio de Utsumi (2000). Una madre le pide a su hijo que ordene su habitación, $\mathrm{y}$, tras comprobar que este ha hecho caso omiso a su petición, pronuncia cualquiera de los siguientes enunciados:
(1)
a. ¡Esta habitación está totalmente ordenada!
b. Esta habitación parece desastrosa.
c. ¿Te importa si te pido, por favor, que arregles tu habitación?
d. Me encantan los niños que ordenan sus habitaciones.

Para que el oyente reconozca estos enunciados como irónicos, Utsumi (2000: 1783) explica que, por un lado, antes de que el hablante los exprese, deben haberse dado tres sucesos, que conforman lo que denomina el entorno irónico: inicialmente, el hablante tiene determinada expectativa (la madre espera que su hijo atienda su petición de ordenar el cuarto); posteriormente, la expectativa del hablante se ve frustrada (la madre comprueba que el niño la desobedece); finalmente, a causa de este choque entre lo que esperaba y lo que realmente es, el hablante tiene una actitud negativa. Por otro lado, para poder identificar e interpretar la ironía, es preciso que los enunciados anteriores muestren implícitamente al oyente que existe dicho entorno irónico. Para ello, tienen que cumplir con todas o alguna de las condiciones del prototipo de la ironía (Utsumi 2000: 1784-1785). En primer lugar, deben aludir a la expectativa del hablante (que el cuarto esté ordenado). En segundo lugar, tienen que ser pragmáticamente insinceros: (1a) incumple la condición de sinceridad del acto de habla aseverativo; (1b) burla la primera máxima de cantidad, pues el verbo parecer implica un

5 De hecho, Jørgensen et alii (1984) muestran que la existencia de una fuente explícita en la que se origina el contenido que se hace eco el enunciado irónico no es condición necesaria para percibir la ironía, sino que simplemente facilita su comprensión, funcionando como un simple mecanismo de llamada de atención al oyente. 
grado de certeza menor del que evidentemente tiene el hablante; (1c) rompe con el principio de cortesía, pues el uso excesivo de fórmulas corteses resulta inadecuado en un contexto familiar; y (1d) es insincero porque el contenido del enunciado se refiere explícitamente a un objeto cuya existencia se presupone (la clase de los niños que son ordenados), pero que no se halla en la realidad del contexto (el hijo no puede adscribirse a dicha clase $)^{6}$. En tercer y último lugar, los enunciados irónicos deben comunicar indirectamente la actitud negativa del hablante hacia la expectativa rota, lo que se consigue mediante el uso de diversos indicadores verbales, como el uso hiperbólico del adverbio totalmente en (1a) o la expresión, mediante el verbo encantar, de una emoción de agrado que resulta contradictoria en el contexto en (1d).

La teoría de la manifestación implícita, aunque es novedosa al construirse a partir de una terminología que no es exclusiva de la pragmática, deja sin resolver cuestiones primordiales para la definición de la ironía. Particularmente, nos planteamos si los enunciados como los del ejemplo (1d), más que irónicos, deberían considerarse lo que comúnmente se conocen como insinuaciones. De acuerdo con Fernández García (2017: 203), el sentido implícito de esta clase de mecanismos surge a partir de la burla de la máxima de cantidad, de modo que "el hablante deja caer sus dardos" a través de una enunciación insuficiente. Esto es justamente lo que ocurre en el ejemplo (1d), pues la madre, al decir que le encantan los niños que ordenan sus habitaciones, presenta como rema una información que es de por sí consabida - es decir, viola la máxima de cantidad—, lo que deja entrever su crítica a la desobediencia del hijo. Asimismo, continúa la confusión entre ironía y enunciados ultracorteses como el de (1c), lo que, como ya resaltamos a propósito de Haverkate (1985), demuestra la inoperatividad de la insinceridad pragmática como criterio delimitador de la ironía.

Por otro lado, Utsumi (2000: 1795) equipara a la víctima de la ironía —el sujeto que está expuesto a la valoración negativa del hablante - con el objeto irónico al que se refiere el contenido del enunciado, pues define a ambos como la persona que intencionalmente impide que la expectativa del hablante se cumpla. El dilema que plantea esta asunción - como, de hecho, señala el propio Utsumi (2000: 1796) - es que un enunciado como ¿Qué tiempo tan agradable! expresado mientras cae una tormenta carecería de víctima, ya que el objeto responsable del revés, el tiempo atmosférico, no es capaz de evaluar como un ataque la crítica del hablante irónico y, por tanto, no puede catalogarse de víctima. El problema se resuelve si se diferencia claramente entre los papeles de víctima, objeto y destinatario de la ironía, de modo que, en el ejemplo citado, sería plausible tanto la ausencia como la existencia de una víctima, si imaginamos que el hablante dirige su ironía a un oyente que, desacertadamente, lo convenció para salir a dar un paseo.

No obstante, la propuesta de Utsumi (2000) presenta algunos aspectos destacables. El primero es su estrategia de definición de la ironía a partir de un prototipo, que nos parece más conveniente, por su flexibilidad, que la de identificar propiedades estables y comunes, ya que permite establecer matices diferenciadores entre las distintas manifestaciones irónicas según el grado en que satisfacen las condiciones del prototipo. El segundo aspecto de la teoría que merece mención es su interés central por atender específicamente al proceso de reconocimiento de la ironía, aunque sus reflexiones al respecto nos resultan muy reduci-

6 Haverkate (1985: 367-369) incluye este tipo de enunciados dentro de los actos de habla aseverativos irónicos. Según el lingüista, aunque su contenido proposicional no es falso, resulta irrelevante al sufrir lo que denomina un proceso de desfocalización referencial, lo que deriva en una interpretación irónica. 
das, al limitarse casi exclusivamente a la intervención de elementos extralingüísticos - los hechos previos a la comunicación y los conocimientos compartidos sobre expectativas personales-, dejando en un segundo plano aquellos relativos a la configuración pragmática del enunciado. En tercer lugar, la condición de que un entorno irónico rodee el proceso de comunicación de la ironía, si bien no nos parece suficientemente válida para explicar dicho mecanismo por enraizar más con motivaciones psicológicas que comunicativas, consideramos que apunta hacia la interesante idea de que lo que activa en el oyente su proceso de reconocimiento y, más aún, lo diferencia de otros fenómenos en los que también se transgrede algún principio pragmático es la especial forma en que el enunciado irónico se interrelaciona con el contexto discursivo. Precisamente, esta reflexión remite nuevamente a Attardo (2000: 817), que concibe la ironía como un enunciado inadecuado en el contexto de ocurrencia, debido a un desajuste pragmático entre el contenido el enunciado y los supuestos contextuales (que conciernen al sistema de valores compartido entre los participantes y, por tanto, implican a sus expectativas). En definitiva, a nuestro modo de ver, la teoría de la manifestación implícita no proporciona una adecuada caracterización pragmática de la ironía en la comunicación porque se fundamenta sobre principios psicológicos más que lingüísticos.

\subsection{La ironía como una inadecuación relevante}

Attardo (2000) retoma los fundamentos de la teoría pragmática tradicional, particularmente los del principio de cooperación de Grice (1975), y enuncia una teoría que aspira a explicar, separadamente, el proceso de reconocimiento y el de interpretación de la ironía verbal. El autor admite que la propuesta griceana original presenta deficiencias, entre otras, que el incumplimiento de una máxima no siempre supone la nulidad del principio de cooperación, y que existen enunciados irónicos que no consisten en decir algo falso. Es por esto por lo que Attardo (2000) decide reelaborar el principio de cooperación, por una parte, introduciendo la supermáxima 'sé adecuado', que engloba a las máximas griceanas originales; y, por otra, formulando el principio de la interrupción mínima posible, más amplio que el de cooperación, que sirve para indicar al oyente que, cuando perciba que su interlocutor está siendo manifiestamente poco cooperativo, debe interpretar favorablemente esa ruptura como el deseo del hablante de ser significativo y, por tanto, tratar de darle sentido dentro del contexto. A partir de estas premisas, Attardo (2000: 817) caracteriza la ironía como un enunciado inadecuado, pues viola una o varias máximas conversacionales y, por extensión, la supermáxima de adecuación, lo que provoca que los supuestos que se derivan de su contenido proposicional no sean compatibles con los supuestos del contexto; y, al mismo tiempo, como un enunciado relevante, ya que, gracias a la acción del principio de la interrupción mínima posible, el oyente es capaz de percibir que existe un motivo por el que el enunciado es deliberadamente inadecuado y, en consecuencia, inferir que responde a una intención comunicativa distinta de la expresada.

El mayor reconocimiento que hacemos a la teoría de la ironía como una inadecuación enunciativa que resulta relevante es que consigue aunar de manera coherente los fundamentos básicos de las perspectivas de análisis más trascendentales dentro del estudio del fenómeno. En lo que respecta a las teorías pragmáticas tradicionales, el propio Attardo (2000: 820) señala que la adecuación contextual se formula en términos parecidos a los que emplea Austin (1962) para definir las condiciones de adecuación de los actos de habla — que, re- 
cordemos, son el elemento central del análisis de Haverkate (1985) y constituyen la base para el desarrollo de la noción de la insinceridad pragmática dentro de las teorías psicolingüísticas, como la de Utsumi (2000)—. Además, la adecuación se combina con el principio de cooperación — de forma que esta incluye al conjunto de las máximas griceanas-. Así, la teoría de la inadecuación relevante supera las deficiencias atribuidas a las teorías pragmáticas estándares, a saber, su incapacidad para distinguir la ironía de otros fenómenos de naturaleza indirecta, debido a su atención exclusiva a las reglas de formación de los actos de habla, pues la adecuación contextual es una noción más amplia, que atañe no solo al nivel ilocutivo, sino a todo lo que rodea la interacción. Por otro lado, Attardo (2000: 821) afirma que su teoría también es compatible con los enfoques psicolingüísticos basados en la alusión a expectativas frustradas, puesto que las condiciones de adecuación y el resto de máximas griceanas que deben cumplirse para adecuarse al contexto cobran sentido en el marco de la comunicación al insertarse dentro de un trasfondo más general referido a las normas y expectativas personales que rigen el comportamiento social. Por último, Attardo (2000: 818) pone en evidencia la importancia de complementar su teoría, de corte neogriceano, con el modelo relevantista de Sperber y Wilson $(1981,1986)$, pues la adecuación y la relevancia son nociones coexistentes, que conciernen a aspectos distintos: mientras que la adecuación, además de depender de factores contextuales, es sensible a criterios de verdad o falsedad, la relevancia está sujeta a relaciones de coherencia. Compartimos esta reflexión, pues solo a partir de la consideración de ambos aspectos pragmáticos es posible explicar que el oyente no tenga inconveniente en aceptar de buena gana que su interlocutor comunique algo falso o incongruente sin ningún reparo.

También cabe destacar que Attardo (2000) es el primero que expresa claramente que el estudio de la ironía debe abordarse conforme a dos centros de interés: el proceso de reconocimiento (qué lleva al oyente a identificar la intención irónica de su interlocutor) y el proceso de interpretación (cómo determina el oyente cuál es el significado discursivo del enunciado irónico). Asimismo, reclama la necesidad de precisar qué elementos caracterizan a la ironía, pero no mediante la búsqueda de rasgos formales, sino a partir de la atención a su valor intencional. Así, Attardo (2001: 118-122) propone una distinción entre indicadores, que son opcionales y sirven para alertar al oyente de la intención irónica (entonación, ciertas expresiones lexicalizadas, elementos kinésicos...), y factores irónicos, cuya aparición es obligatoria, pues son los que consiguen que determinado enunciado adquiera una interpretación irónica (formas variadas de provocar un desajuste, bien entre elementos relativos al propio comportamiento comunicativo del hablante, bien entre el enunciado y el contexto).

En resumen, Attardo (2000) presenta un modelo teórico que es abarcador y cuyos fundamentos están a priori libres de contradicciones - a falta de comprobar su validez aplicándolos en casos reales - Sin embargo, admitimos que no es suficiente para definir la ironía. De hecho, la mayor objeción que hacemos a la teoría es que no ofrece una descripción pormenorizada acerca de en qué consiste el sentido irónico, refiriéndose a él simplemente como "an antiphrastic meaning (i.e. in the direction of the opposite of what [speaker] is saying" (Attardo 2000: 816). Opinamos que se trata de una explicación del modo en que se interpreta la ironía manifiestamente inservible, no solo por su brevedad y vaguedad, sino por su naturaleza semántica, que frena las aspiraciones de Attardo de construir un modelo explicativo que sea aplicable a los actos de habla irónicos realizativos, cuyo significado 
discursivo no es una realidad contraria u opuesta, sino un objetivo comunicativo distinto al que se consigue por medio del enunciado, o la expresión del deseo del hablante de no alcanzarlo — tal y como señala Haverkate (1985)—.

\subsection{La ironía como la transgresión de los principios conversacionales levinsonianos}

El grupo GRIALE propone en una serie de trabajos, recogidos en su mayoría en Ruiz Gurillo y Padilla, eds. (2009), un nuevo modelo de análisis de enfoque neogriceano, que se fundamenta sobre los principios de Levinson (2000) y que trata de explicar la ironía a partir de la observación e identificación de ciertos patrones de comportamiento lingüísticos que son recurrentes en ella e independientes del contexto particular en que se produce. Así, la ironía se postula como una implicatura conversacional particularizada que surge por la infracción intencional del requisito previo de cualidad, lo que provoca la inversión del funcionamiento del resto de principios pragmáticos —el de cantidad, el de manera y el de informatividad-, de modo que determinados elementos lingüísticos presentes en el enunciado, los denominados indicadores irónicos, ven alterado su significado "recto" para adquirir una interpretación irónica. Es decir, los indicadores se conciben como estructuras que, en cierto modo, contienen un valor irónico codificado en ellos mismos, de ahí que su empleo sirva "como detonante de una interpretación marcada por parte del oyente" (Padilla 2009: 148).

Cuando el principio afectado es el de cantidad ${ }^{7}$, entonces se niega el valor escalar de los cuantificadores, los superlativos y ciertos sufijos y prefijos, y, al mismo tiempo, se afirma el valor del miembro más fuerte. Para explicarlo, Provencio (2009: 250) presenta una conversación que mantienen dos amigas sobre las palabras que escucharon a un tercero:

(2)

A: y le oigo que le decía a Javi Marta está gordita.

B: ya, gordita. ¡Qué víbora!

El empleo irónico del sufijo -ita supone la negación de su valor primario de aminoración de la cualidad de estar gorda, así como la implicación del valor opuesto, el de aumento ('Marta está obesa'), y de un valor intensificador-despectivo que contradice al atenuador-afectivo original. Si se invierte el principio de manera ${ }^{8}$, el sentido irónico se localiza en las expresiones marcadas que contiene el enunciado, como ciertas figuras retóricas, unidades fraseológicas o recursos propios de una variedad diafásica distinta de la que impone la situación comunicativa concreta. Así ocurre, según ilustra Rodríguez Rosique (2009: 123), en el ejemplo (3), donde el hablante, a pesar de encontrarse en una situación informal, se refiere a su profesión con un tecnicismo burocrático en lugar de con una palabra más corriente, como transportista:

(3) Pues agente de servicios auxiliares. Cargando y descargando.

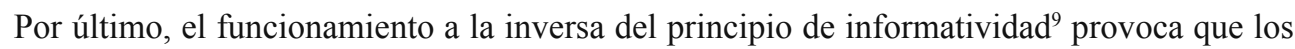
referentes se multipliquen y den paso a los dobles sentidos, de ahí que repercuta en pala-

7 'No proporcione una información más débil que el conocimiento del mundo que posee'.

8 'Indique una situación normal mediante expresiones no marcadas'.

9 'Proporcione información mínima que sea suficiente para conseguir sus propósitos comunicativos'. 
bras polisémicas u homónimas, entre otras. Rodríguez Rosique (2009: 122) lo ejemplifica a través de una conversación en la que, a propósito de los problemas de corazón que padece el padre de uno de los participantes, otro interlocutor dice:

(4) Yo también tengo problemas de corazón. Lo tengo muy tierno.

Junto a los indicadores, también se encuentran una serie de marcas, que se diferencian de los primeros en que no encierran un valor irónico, sino procedimental, pues sirven para alertar al oyente de que el hablante infringe el requisito previo de cualidad y que, por ende, debe reconocer el enunciado como irónico. Además de marcas acústico-melódicas — que son las más características y que configuran lo que Padilla (2009: 149) denomina el tono irónico-, existe una amplia gama de marcas paralingüísticas, kinésicas y proxémicas (Cestero 2009) — de naturaleza no verbal, que pueden reforzar el carácter irónico del enunciado-, así como otras marcas verbales, como los evidenciales — que, empleados irónicamente, invierten o destruyen la información modal que precisan-. Es el caso de (5), en el que un hablante, refiriéndose a su familia, dice:

(5) Con mi familia... con mi familia me llevo bastante bien. Tengo una hermana... y padres. Eso... eso dicen.

De acuerdo con Santamaría (2009: 282), el hablante, mediante el evidencial eso dicen, pone en duda la paternidad de sus padres, lo que resulta evidentemente falso al contrastar dicha información con el contexto sociocultural - los saberes compartidos entre los participantes-, pues el interlocutor sabe de antemano que el hablante tiene la certeza de quiénes son sus progenitores. Así, el oyente interpreta irónicamente que lo que se asume como algo incierto, en realidad, es evidente y seguro.

Desde nuestro punto de vista, la propuesta neogriceana destaca notoriamente sobre los estudios anteriores porque sus principios teóricos se construyen —a la vez que se validan - con base en un análisis sistemático de usos lingüísticos reales extraídos de diversos corpus orales y escritos en español. Este rigor metodológico permite desarrollar ideas nuevas o apenas esbozadas hasta entonces, como la gramaticalización del sentido irónico en algunas unidades fraseológicas (Ruiz Gurillo 2009), la existencia de una ironía focalizada (vinculada al contexto situacional y lingüístico inmediato) y otra continuada (originada en los conocimientos compartidos) (Reus Boyd-Swan 2009), o la clasificación de la ironía en positiva o negativa según sus efectos (des)corteses (Alvarado 2009) ${ }^{10}$. Igualmente, reconocemos el empeño de GRIALE por ofrecer una teoría que se vincule con principios propiamente pragmáticos, que explique tanto los casos prototípicos, consistentes en un significado contrario (por inversión del principio de cantidad), como los que encierran un significado distinto (cuando la inversión implica a los principios de manera e

10 Aunque alabamos el interés por analizar las relaciones entre ironía y (des)cortesía verbal, hemos de puntualizar que las conclusiones del estudio nos parecen reducidas, pues presentan los efectos corteses o descorteses de la ironía como correlativos a una actitud positiva o negativa del hablante hacia el objeto irónico. En nuestra opinión, los posibles efectos (des)corteses se generan en función del tipo de vínculo que el oyente mantenga con el objeto sometido a la valoración irónica (no de la orientación positiva o negativa del acto valorativo). Esto explica que, en ocasiones, una ironía negativa sobre una persona ausente sirva como estrategia cortés para congraciarse con el oyente si este tampoco siente agrado por el objeto de la ironía. 
informatividad), y que trate de dar una respuesta, respectivamente, a la cuestión de cómo se reconoce y cómo se interpreta la ironía.

Sin embargo, detectamos una debilidad esencial: la ruptura del requisito de cualidad no sirve como criterio delimitador de la ironía frente a otros conceptos relacionados (figuras retóricas o los juegos de palabras), de los que también puede decirse que se invierte la máxima de cualidad, además del resto de principios levinsonianos. De hecho, tal deficiencia teórica lleva a los investigadores a analizar como irónicos ejemplos que no lo son. Es el caso de (3), que, si bien explota el principio de manera para, en conjunción con los condicionantes contextuales, producir efectos humorísticos, no puede afirmarse que sea irónico, pues no supone una negación de sentido (la profesión es la misma independientemente de si te refieres a ella con un término habitual o con uno técnico). Igualmente, el ejemplo (4) consiste en un juego de palabras entre las dos acepciones del sustantivo corazón (como órgano físico y como sentimientos), que, según nuestras apreciaciones, surge porque la contribución del hablante no se relaciona con el propósito de la comunicación (los problemas de salud físicos). Aunque la información aportada es irrelevante, no es falsa, pues, presuponemos, se da el hecho de que el hablante es ciertamente una persona afectuosa, "de corazón tierno", y todos los participantes comparten esa idea. Distinto sería si el hablante se describiera a sí mismo como una persona sensible cuando tanto él como el resto saben que, en realidad, es una persona despegada y distante. Entonces, el empleo de un referente que no puede adecuarse a los supuestos del contexto provocaría el surgimiento de una interpretación irónica.

Por otro lado, el modelo neogriceano, al establecer que la transgresión del requisito de cualidad desencadena una serie de "consecuencias semánticas" sobre determinados elementos lingüísticos (Rodríguez Rosique 2009: 120), excluye de su análisis todos aquellos actos de habla irónicos distintos de los asertivos, que no pueden considerarse falsos, sino fallidos, debido a que no cumplen con la condición necesaria para que su realización se considere adecuada, de modo que su sentido irónico consiste en la inversión no del significado de un lexema concreto, sino del valor de su fuerza ilocutiva - recordemos los postulados de Haverkate (1985), que muestran, por ejemplo, cómo en un acto directivo la ironía se manifiesta como la negación de la fuerza ilocutiva-. Asimismo, la condición de la infracción de la cualidad no es un criterio operativo para justificar los casos en los que la ironía no afecta al significado proposicional, sino a la modalidad del enunciado, como ocurre en (5). Aquí, el sentido irónico no se revela como un significado opuesto del explícito (*ddicen que no tengo una hermana y padres'), sino como una inversión o negación del grado de certeza que comunica el hablante con respecto a lo dicho ('digo que no sé a ciencia cierta si mi familia es la biológica, cuando, en realidad, sí estoy seguro de ello'). No obstante, hemos de alabar que el modelo neogriceano es pionero en sugerir la existencia de esta clase de ironía radicada en el nivel modal.

En definitiva, la crítica fundamental que hacemos al modelo neogriceano es que no ofrece un criterio que determine inequívocamente cómo se reconoce e interpreta la ironía, y que, además, incluya los casos que consisten en algo más que una inversión semántica. Creemos que las expresiones lingüísticas que GRIALE cataloga de indicadores no son, como afirma Padilla (2009: 148), las "que suministra[n] ironía al enunciado de manera explícita", sino que, en todo caso, contienen el sentido irónico, si bien este se activa por acción de otro elemento — que, según observamos en los ejemplos de sus trabajos, parece ser la inadecuación contextual- 


\section{DISCUSIÓN}

La revisión bibliográfica presentada pone en evidencia, en primer lugar, que no existe una teoría aplicable a la totalidad de manifestaciones irónicas posibles, ya que sus fundamentos solo alcanzan a explicar satisfactoriamente los casos más prototípicos, en los que el sentido irónico radica en el nivel proposicional del enunciado, dejando al margen aquellos que atañen a la fuerza ilocutiva o a la modalidad del enunciado. En segundo lugar, las diversas propuestas no proporcionan una explicación unánime acerca del modo en que se reconoce el sentido irónico, encontrando discrepancias entre las que lo fundamentan porque el oyente percibe que el hablante es insincero - ya sea con respecto a la verdad de lo que dice, ya sea en lo relativo a sus intenciones comunicativas - o expresa su actitud negativa ante una opinión o expectativa, o detecta que la enunciación es inadecuada en el contexto. A esto hay que añadir, en tercer lugar, que la mayoría de los estudios no aborda separadamente la etapa del reconocimiento y de la interpretación, lo que también contribuye a la fragmentariedad de sus reflexiones. En cuarto lugar, los modelos establecen unos criterios de caracterización que son por sí solos insuficientes para delimitar nítidamente el fenómeno irónico frente a otros que también son de naturaleza implícita, como las peticiones indirectas, los enunciados ultracorteses o las insinuaciones. Por último, ofrecen una visión simplista tanto de los motivos para el uso de la ironía como de los efectos que provoca en el plano social, que se reducen, respectivamente, a la crítica y a la ofensa, lo que, en parte, viene motivado por el fallo a la hora de diferenciar los conceptos de víctima, objeto y destinatario de la ironía. Numerosas teorías equiparan al objeto de la ironía con la víctima de la valoración irónica, declaración que resulta desacertada porque implica presuponer que la ironía entraña siempre un juicio negativo y que, por tanto, siempre victimiza al objeto irónico; otras no distinguen entre objeto y destinatario, de modo que asumen erróneamente que la valoración que expresa el enunciado sobre el objeto irónico se dirige siempre al destinatario, normalmente a modo de ataque.

Para confirmar o refutar estas conclusiones, confrontaremos los modelos teóricos analizados con algunos casos de ironías procedentes del corpus que mencionábamos al final del primer epígrafe de este trabajo, más concretamente, de debates y entrevistas del programa televisivo de La Sexta Noche. Comencemos considerando el ejemplo (6), tomado de un debate. El periodista Antonio Maestre acusa a La Falange de convertir los monumentos conservados de la época franquista en lugares de peregrinación fascista. María Claver, adversaria ideológica, le replica que, cuando hable de La Falange "como si fuera una cosa de extrema derecha", tenga en cuenta que en ella militaron muchos que posteriormente formaron parte de partidos de izquierdas. Maestre le responde irónicamente:

(6) No, no, no es de extrema derecha. La Falange no es de extrema derecha. Seguid con el revisionismo.

Siguiendo el análisis de Haverkate (1985), en este fragmento nos encontramos, en primer lugar, con un acto de habla asertivo que, al romper la condición de sinceridad —el hablante no cree realmente en la verdad de lo dicho--, implica un significado contrario ('La Falange sí es de extrema derecha'); y, en segundo lugar, con un acto de habla directivo (una petición) que tampoco cumple la condición de sinceridad —el hablante no desea hacer que su 
interlocutora continúe replanteando las doctrina política del franquismo-, lo que deriva en la negación de su fuerza ilocutiva ('no sigáis con el revisionismo'). Ahora bien, Haverkate (1985) sostiene que la expresión de una cortesía fingida también puede llevar a una interpretación irónica. Pensemos que el hablante irónico hubiera dicho Por favor, seguid con el revisionismo si sois tan amables. Es verdad que un uso ultracortés provocaría que los efectos potencialmente corteses de las fórmulas empleadas se cancelasen e invirtiesen - tornándose descorteses - Sin embargo, esta clase de insinceridad retórica no determina la interpretación irónica del enunciado — con la consecuente transformación de la fuerza ilocutiva-, pues, como vemos, esta surge también en (6), donde no existe un desajuste en el grado de cortesía expresado. Por consiguiente, la insinceridad de la ironía resulta únicamente del incumplimiento de la condición de sinceridad del acto de habla, no de la ruptura de una convención de cortesía.

Si atendemos a los fundamentos de la teoría de la mención ecoica (Sperber y Wilson 1981, 1986), puede afirmarse que el contenido de (6) se hace eco — casi de forma exactade las opiniones de la interlocutora, para así manifestar el hablante su actitud negativa hacia ellas - y contradecirlas- . Lo mismo ocurre en el ejemplo (7), extraído de otro debate. Jesús Maraña aduce que uno de los motivos principales por los que se ha suspendido el campeonato de vela Barcelona World Race es que, a pocas semanas del inicio, "una orden en el BOE modifica las exenciones fiscales para las empresas patrocinadoras". Francisco Marhuenda, debatiente afín al gobierno — por entonces, del Partido Popular-, que, además, ha defendido previamente que la suspensión se debe exclusivamente a la complicada situación política de Cataluña, a raíz del movimiento independentista, responde:

(7) Sí. Es culpa de Rajoy. Está claro.

El hablante menciona un contenido que se asemeja proposicionalmente a lo dicho por su interlocutor, ya que Mariano Rajoy, como presidente del gobierno, es quien, en última instancia, aprueba las disposiciones del BOE, por lo que las consecuencias que estas tengan son su responsabilidad. De este modo, Marhuenda expresa su rechazo al argumento de Maraña.

Observemos ahora el enunciado (8), extraído de una entrevista a Boris Izaguirre. Este se dispone a juzgar unas imágenes de la familia real mientras toman su almuerzo diario y advierte de antemano que "le choca criticar a la reina", pues se considera promonárquico. A continuación, pronuncia un discurso cargado de críticas hacia todo lo que rodea la escena - desde la comida, pasando por los utensilios de la mesa, hasta la decoración de la estancia—. Cuando finaliza, Xavier Sardà, colaborador del programa, le dice entre risas:

(8) Menos mal que no querías criticar a la reina.

Claramente, alude a las palabras previas del interlocutor para poner en evidencia lo contradictorias que resultan en vista de sus acciones. Sin embargo, no se trata de una ironía, pues Sardà no implica lo opuesto ('no siento alivio de que no quisieras criticar a la reina' o 'menos mal que querías criticar a la reina'), sino que más bien da a entender que, efectivamente, es un alivio que Izaguirre sienta respeto hacia la monarca, pues no imagina cómo de incisivos habrían sido sus comentarios si no hubiera sido así. 
Por tanto, la presencia de un eco no es una propiedad exclusiva de la ironía. De hecho, ni siquiera puede afirmarse que todos los enunciados irónicos mencionen un contenido - expresado anteriormente o atribuido a alguien_-, como se demuestra en el caso (9). En un debate sobre si se debe o no poner límites a la libertad de expresión, María Claver lee en voz alta fragmentos de una de las canciones del rapero Valtònyc, condenado a varios años de prisión por sus letras violentas y amenazantes, para evidenciar la necesidad de perseguir este tipo de actos. Elisa Beni, contraria a las ideas de Claver, interrumpe su intervención para preguntarle con sorna por qué ella puede leer tranquilamente la canción y su autor no puede cantarla, a lo que Claver responde:

(9) El límite, el límite no lo pongo yo, querida, lo ha puesto la Audiencia Nacional. Y lo ha puesto el Tribunal Supremo. Yo sé que eres una gran jurista... que yo sé que... que yo sé que tú eres una gran jurista, pero indiscutiblemente ha habido una Audiencia Nacional y ha habido un Tribunal Supremo que ha ratificado esa condena.

Resulta imposible identificar alguna fuente explícita previa de la que se haga eco el contenido del enunciado irónico. Y tampoco es probable que el eco sea implícito y aluda a una supuesta creencia de Beni acerca de su enorme profesionalidad como jurista, ya que no es esa su profesión (es periodista).

Asimismo, encontramos casos como (10) en los que, además de no haber mención ecoica, tampoco hay una actitud negativa. En una entrevista a las actrices Cristina Castaño y Ana Morgade, el entrevistador Iñaki López recalca en repetidas ocasiones el gran parecido que existe entre la trama argumental de una serie televisiva que acaban de grabar y la realidad política española. Una de las veces, Morgade añade:

(10) Pero es totalmente anecdótico. No está basado en la realidad super para nada. No tiene nada que ver.

En este caso la ironía no sirve al hablante para marcar su desacuerdo con algo dicho por el oyente, pues, como indicamos, este explicita previamente exactamente lo mismo que implica el enunciado irónico: que la serie se basa en hechos reales. Nos encontramos ante un ejemplo de ironía en el que el ironista, más que una actitud negativa, adopta una que podríamos calificar de expresiva, de llamada de atención sobre el objeto irónico, que le permite reforzar lazos con el interlocutor al mostrarle que se sitúa en la misma posición ideológica. Por tanto, concluimos, por un lado, que la mención ecoica, si bien es un uso lingüístico que puede aparecer en la ironía cuando se pretende criticar la opinión del oyente, ni es permanente ni es exclusiva del fenómeno, y, por otro lado, que la expresión de una actitud negativa tampoco es una característica definitoria de la ironía, pues no ocurre siempre.

Continuamos el análisis aplicando las condiciones del prototipo de la ironía propuestas por Utsumi (2000). Si volvemos al ejemplo (9), en el que María Claver irónicamente califica a Elisa Beni de gran jurista, podría afirmarse que el contenido del enunciado, en cierto modo, alude a una expectativa rota del hablante, a saber, que Beni hubiese construido sus argumentos según los fundamentos del derecho y no a partir de apreciaciones subjetivas injustificadas. Asimismo, Claver muestra una actitud negativa hacia ese deseo frustrado por Beni, de modo que esta se convierte en la víctima de la ironía. Y, por último, el enunciado es un acto asertivo que rompe la condición de sinceridad, por lo que se implica un sentido 
negado ('no eres una gran jurista'). Sin embargo, el modelo del prototipo irónico no es aplicable en el caso (10). En primer lugar, la condición de la alusión a una expectativa frustrada del hablante no se cumple, pues Morgade en ningún momento previo a la expresión del enunciado declara o da a entender que esperase que el argumento de la serie fuera pura invención —es decir, no hay un entorno irónico - En segundo lugar - como ya hemos señalado-, el hablante, más que expresar una actitud negativa, busca enfatizar que, efectivamente, el parecido de la trama con la vida real no es casual. Por tanto, tampoco hay una víctima — un culpable de que el hablante no pueda cumplir sus deseos - ${ }^{11}$, simplemente un objeto irónico (el argumento de la serie). La única condición del prototipo irónico que cumple (10) es la referida a la insinceridad pragmática, pues ninguno de los actos asertivos es sincero, de ahí que su significado literal se interprete como lo contrario ('es intencionado' y 'sí tiene mucho que ver con la realidad'). Por tanto, la teoría de la manifestación implícita solo acierta al considerar la ironía como un enunciado que rompe la condición de sinceridad del acto de habla — lo que va en consonancia con los planteamientos de Haverkate (1985) -

Por lo que respecta al modelo neogriceano de GRIALE (Ruiz Gurillo y Padilla, eds. 2009), el requisito previo de la transgresión de la cualidad no se da en el caso de (6), donde - recordemos - Antonio Maestre insta irónicamente a María Claver a que continúe con el revisionismo. Al tratarse de un acto de habla distinto del asertivo, no nos encontramos ante un contenido proposicional falso, sino ante una fuerza ilocutiva insincera, ya que no se interpreta como un intento del hablante de hacer que el oyente realice cierto acto futuro. El requisito de la cualidad tampoco es aplicable en el ejemplo (11), expresado durante una entrevista al periodista Arcadi Espada. El entrevistador Iñaki López pregunta a Espada qué opina sobre la llamada que Francisco Camps — expresidente de la Generalitat valenciana- hizo a Juan Luis de la Rúa, juez del Tribunal Superior de Justicia de la Comunidad Valenciana, tras ser aquel imputado por varios delitos de corrupción. Espada explica que dicha llamada fue fruto del enfado de Camps al considerar que estaba recibiendo un trato discriminatorio por parte de la justicia. Iñaki López rebate que ni él ni cualquier otro ciudadano corriente tienen la potestad para llamar personalmente a un juez y exponerle sus quejas por una condena, a lo que Espada responde que, obviamente, no pueden, porque no son el presidente de la Comunidad Valenciana. Entonces, Iñaki replica:

(11) ¡Ah! Yo creía que éramos todos iguales.

Aquí la ironía no radica en el nivel proposicional —en el significado léxico-, sino en el modal, esto es, en el grado de certeza del hablante sobre lo dicho. El entrevistador, mediante el empleo de un predicado doxástico (creer), manifiesta que no está totalmente seguro de la imparcialidad de la justicia, cuando, en realidad, este es un principio asumido e indiscutible. El hablante asevera un hecho que es semánticamente verdadero - por lo que no hay burla de la máxima de cualidad-, si bien expresa un grado de compromiso con respecto a la verdad de la proposición que es más débil del que se le presupone por el saber del mundo compartido - viola la máxima de cantidad-. Este desajuste contextual deriva en una inversión de la modalidad — de lo incierto a lo seguro_- que en (11) se marcaría lingüísticamente por medio de un predicado epistémico como saber.

11 Es más, si se diera el caso de que el hablante mostrase una actitud negativa, no parece lógico afirmar que el supuesto culpable - y víctima de la ironía - sea un objeto inanimado como es la serie. 
Acabamos el análisis con la teoría de Attardo (2000), que postula que la ironía se reconoce porque el enunciado es contextualmente inadecuado. Si tomamos los ejemplos irónicos anteriores, observamos que el contenido de todos ellos choca con el bagaje presuposicional. En (6) lo aseverado (La Falange no es de extrema derecha) es incompatible con el conocimiento general (la historia política de España), y la acción que el hablante pide al oyente (que siga opinando) es incongruente con los supuestos que se derivan del discurso anterior de aquel (que su opinión es inadmisible); en (7) lo aseverado (Rajoy es el culpable de la cancelación de la competición de vela) entra en contradicción con lo dicho previamente por el hablante (la causa ha sido la crisis política de Cataluña), además de con su identidad (es miembro del mismo partido político que Rajoy); en (9) la atribución que se hace al oyente (eres una gran jurista) es incompatible con su identidad (es periodista); en (10) lo aseverado (la serie es totalmente ficticia) contradice las informaciones dadas previamente (la trama caricaturiza la realidad); y en (11) la actitud de incertidumbre que expresa el hablante sobre la ocurrencia de cierto hecho (la igualdad jurídica) choca con la seguridad que, por el saber del mundo compartido, se asume que tiene al respecto. Por consiguiente, la existencia de un desajuste entre el enunciado irónico y las informaciones que se derivan del contexto es una propiedad que, además de ser común a toda clase de ironías, funciona como filtro para su reconocimiento — tal y como sostiene Attardo (2000)-.

\section{CONCLUSIONES}

A lo largo de estas páginas hemos reflexionado acerca de las debilidades y fortalezas de cinco de las teorías que más trascendencia han tenido en el estudio de la ironía verbal. Tras una primera parte de revisión crítica bibliográfica, encaminada, por un lado, a identificar las deficiencias de los modelos y, por otro, a aislar posibles fundamentos válidos, hemos continuado con una segunda parte de confrontación de las teorías con las muestras de un corpus oral, en aras de comprobar o refutar las conclusiones extraídas en la parte anterior. Así, de las cinco, solo los presupuestos del análisis pragmalingüístico de Haverkate (1985) y de la teoría de la inadecuación relevante de Attardo (2000) han sido aplicables a todos los casos irónicos analizados. Los resultados muestran que, como afirman Colston y Gibbs (2007: 4), aunque las teorías son múltiples, no son incompatibles entre ellas, sino que "each offer a different perspective on the phenomenon". Por ello, por la coherencia y amplitud de sus fundamentos, los dos modelos señalados constituyen una base idónea para lograr aproximarnos a una definición de la ironía que sea válida y lo más inclusiva posible.

Por lo que respecta al proceso de reconocimiento, Attardo (2000) parece ofrecer la clave para explicar cómo se activa: por el desajuste entre el enunciado y el contexto, porque las presuposiciones que se derivan del contenido proposicional no coinciden con el sistema de conocimientos y creencias que el oyente comparte con el hablante, de modo que el enunciado transgrede alguna de las condiciones de adecuación del acto de habla. Así, por acción del principio de relevancia, el oyente infiere que la inadecuación enunciativa tiene determinado valor comunicativo en el contexto. En nuestra opinión, la inadecuación contextual tiene altas posibilidades de postularse como una propiedad específica de la ironía, que la distinguiría de otros mecanismos no literales que, a pesar de burlar algún principio pragmático, no se perciben como un uso incongruente con el mundo extralingüístico. 
En cuanto al proceso de interpretación, los datos manejados en los diversos trabajos parecen corroborar la idea de Haverkate (1985) de que el sentido irónico puede localizarse, bien en el nivel proposicional — en los actos de habla asertivos-, generándose una implicatura que deriva en un significado que niega o se opone al literal; bien en el ilocutivo - en el resto de actos de habla-, lo que resulta en la negación de su fuerza ilocutiva o en el surgimiento de otra distinta, manifestándose entonces la ironía como un acto de habla indirecto. Además, como ya apuntan Sperber y Wilson $(1981,1986)$ y confirman Ruiz Gurillo y Padilla, eds. (2009), hay que incluir un tipo de ironía que también se da en los enunciados asertivos, si bien no radica en el nivel semántico, sino en el modal, de ahí que el contenido no pueda calificarse estrictamente como falso.

Finalmente, a falta de un estudio pormenorizado que atienda a las funciones y efectos de la ironía, los datos apuntan a que, a diferencia de lo defendido por la mayoría de los autores, la ironía expresa una actitud del hablante que puede tener una orientación tanto negativa como positiva, pasando por una expresiva de naturaleza humorística. No obstante, siguiendo la línea marcada por Sperber y Wilson $(1981,1986)$, en ocasiones la ironía puede expresarse formalmente por medio de una mención ecoica del discurso previo del interlocutor, para, además de expresar una actitud negativa, llevar a cabo otras estrategias retórico-argumentativas específicas, como presentar una idea como absurda $\mathrm{y}$, de este modo, contradecir al interlocutor y mostrar su inferioridad. Asimismo, la ironía puede generar efectos variados en el plano social, que van de lo cortés — por ejemplo, generar complicidad con el interlocutor, en aras de estrechar lazos con él—a lo descortés — atacarlo a través de la crítica irónica-

Las observaciones al respecto se constituyen como el punto de partida para el estudio de la ironía verbal, pues no solo dan cuenta de las cuestiones aún por resolver, sino que orientan sobre el modo de abordarlas. El trabajo de investigación de mayor envergadura en el que esta publicación se incardina continúa con la aplicación sistemática de las bases teóricas que aquí identificamos como potencialmente válidas en las muestras de nuestro corpus, de modo que corroboremos su idoneidad, presentando sus resultados más significativos en De la Casa (en preparación).

\section{REFERENCIAS BIBLIOGRÁFICAS}

Alba Juez, L. (1995). "Verbal irony and the Maxims of Grice's Cooperative Principle", Revista Alicantina de Estudios Ingleses, 8, pp. 25-30. http://dx.doi.org/10.14198/raei.1995.8.02

Alvarado Ortega, M. B. (2009). "Ironía y cortesía". En Ruiz Gurillo, L. y X. A. Padilla García (eds.). Dime cómo ironizas y te diré quién eres. Una aproximación pragmática a la ironía. Frankfurt: Peter Lang, pp. 333-346.

Attardo, S. (2000). "Irony as relevant inappropriateness", Journal of Pragmatics, 32 (6), pp. 793-826. https://doi.org/10.1016/S0378-2166(99)00070-3

Attardo, S. (2001). Humorous texts: a semantic and pragmatic analysis. Berlín/Nueva York: Mouton de Gruyter.

Austin, J. L. (1962). Cómo hacer cosas con palabras: palabras y acciones. Barcelona: Paidós, 2010.

Barbe, K. (1995). Irony in Context. Amsterdam: John Benjamins.

Bruzos Moro, A. (2006). "Sobre el problemático concepto de mención irónica", ELUA: Estudios de Lingüistica. Universidad de Alicante, 20, pp. 33-55. https://doi.org/10.14198/ELUA2006.20.02

De la Casa, L. (en preparación). "Reconocimiento e interpretación: dos fases en el análisis de la ironía". 
Cestero Mancera, A. M. (2009). "Marcas paralingüísticas y kinésicas de la ironía”. En Ruiz Gurillo, L. y X. A. Padilla García (eds.). Dime cómo ironizas y te diré quién eres. Una aproximación pragmática a la ironía. Frankfurt: Peter Lang, pp. 167-190.

Clark, H. y R. Gerrig (1984). "On the pretense theory of irony", Journal of Experimental Psychology: General, 113, pp. 121-126.

Colston, H. L. y R. W. Gibbs (2007). "A Brief History of Irony”. En Gibbs, R. W. y H. L. Colston (eds.). Irony in Language and Thought: A Cognitive Science Reader. Nueva York: Lawrence Erlbaum Associates, pp. 3-21.

Ducrot, O. (1984). El decir y lo dicho. Barcelona: Paidós, 1986.

Fernández García, F. (2017). La descortesía en el debate electoral cara a cara. Sevilla: Editorial Universidad de Sevilla (Colección Lingüística).

Gibbs, R. W. y H. L. Colston (eds.) (2007). Irony in Language and Thought: A Cognitive Science Reader. Nueva York: Lawrence Erlbaum Associates.

Gibbs, R. W. y J. O'Brien (1991). "Psychological aspects of irony understanding", Journal of Pragmatics, 16 (6), pp. 523-530. https://doi.org/10.1016/0378-2166(91)90101-3

Giora, R. (1995). "On Irony and Negation”, Discourse Processes, 19 (2), pp. 239-264. https://doi. org/10.1080/01638539509544916

Giora, R. (1997). "Understanding figurative and literal language: The graded salience hypothesis", Cognitive Linguistics, 7, pp. 183-206.

Grice, H. P. (1975). "Logic and Conversation". En Cole, P. y J. L. Morgan (eds.). Syntax and Semantics. Vol. 3: Speech Acts. Nueva York: Academic Press, pp. 41-58.

Grice, H. P. (1978). "Further notes on logic and conversation". En Cole, P. (ed.). Syntax and Semantics. Vol. 9: Pragmatics. Nueva York: Academic Press, pp. 113-127.

Haverkate, H. (1985). "La ironía verbal: un análisis pragmalingüístico", Revista Española de Lingüística, 15, pp. 343-391. http://revista.sel.edu.es/index.php/revista/article/view/1077

Jørgensen, J., G. Miller y D. Sperber (1984). "Test of the mention theory of irony", Journal of Experimental Psychology: General, 113, pp. 112-120.

Kumon-Nakamura, S., S. Glucksberg y M. Brown (1995). "How about another piece of pie: the allusional pretense theory of discourse irony". En Gibbs, R. W. y H. L. Colston (eds.). Irony in Language and Thought: A Cognitive Science Reader. Nueva York: Lawrence Erlbaum Associates, pp. 57-95, 2007.

Levinson, S. C. (2000). Presumptive Meaning. The Theory of Generalized Conversational Implicature. Cambridge/Massachusetts: The MIT Press.

Littman, D. y J. Mey (1991). "The nature of irony: Toward a computational model of irony”, Journal of Pragmatics, 15 (2), pp. 131-151. https://doi.org/10.1016/0378-2166(91)90057-5

Marimón Llorca, C. (2009). "La retórica". En Ruiz Gurillo, L. y X. A. Padilla García (eds.). Dime cómo ironizas y te diré quién eres. Una aproximación pragmática a la ironía. Frankfurt: Peter Lang, pp. 13-44.

Padilla García, X. A. (2009). "Marcas acústico-melódicas: el tono irónico". En Ruiz Gurillo, L. y X. A. Padilla García (eds.). Dime cómo ironizas y te diré quién eres. Una aproximación pragmática a la ironia. Frankfurt: Peter Lang, pp. 135-166.

Provencio Garrigós, H. (2009). "La prefijación y la sufijación”. En Ruiz Gurillo, L. y X. A. Padilla García (eds.). Dime cómo ironizas y te diré quién eres. Una aproximación pragmática a la ironía. Frankfurt: Peter Lang, pp. 241-265.

Reus Boyd-Swan, F. (2009). "Cómo se manifiesta la ironía en un texto escrito". En Ruiz Gurillo, L. y X. A. Padilla García (eds.). Dime cómo ironizas y te diré quién eres. Una aproximación pragmática a la ironía. Frankfurt: Peter Lang, pp. 293-305.

Rodríguez Rosique, S. (2009). "Una propuesta neogriceana". En Ruiz Gurillo, L. y X. A. Padilla García (eds.). Dime cómo ironizas y te diré quién eres. Una aproximación pragmática a la ironía. Frankfurt: Peter Lang, pp. 109-132. 
Ruiz Gurillo, L. (2009). "La gramaticalización de unidades fraseológicas irónicas". En Ruiz Gurillo, L. y X. A. Padilla García (eds.). Dime cómo ironizas y te diré quién eres. Una aproximación pragmática a la ironía. Frankfurt: Peter Lang, pp. 371-390.

Ruiz Gurillo, L. (2010). "Para una aproximación neogriceana a la ironía en español”, RSEL: Revista Española de Lingüística, 40 (2), pp. 95-124. http://revista.sel.edu.es/index.php/revista/article/ view/87

Ruiz Gurillo, L. y X. A. Padilla García (eds.) (2009). Dime cómo ironizas y te diré quién eres. Una aproximación pragmática a la ironía. Frankfurt: Peter Lang.

Santamaría Pérez, I. (2009). "Los evidenciales". En Ruiz Gurillo, L. y X. A. Padilla García (eds.). Dime cómo ironizas y te diré quién eres. Una aproximación pragmática a la ironía. Frankfurt: Peter Lang, pp. 267-292.

Searle, J. (1969). Actos de habla. Ensayo de filosofia del lenguaje. Madrid: Ediciones Cátedra, 2001.

Sperber, D. y D. Wilson (1981). "Irony and the Use-mention Distinction". En Cole, P. (ed.). Radical Pragmatics. Nueva York: Academic Press, pp. 295-317.

Sperber, D. y D. Wilson (1986). Relevance. Communication and Cognition. Oxford: Blackwell, 1995.

Torres Sánchez, M. A. (1999). Aproximación pragmática a la ironía verbal. Cádiz: Servicio de Publicaciones de la Universidad de Cádiz.

Torres Sánchez, M. A. (2009). "La relevancia". En Ruiz Gurillo, L. y X. A. Padilla García (eds.). Dime cómo ironizas y te diré quién eres. Una aproximación pragmática a la ironía. Frankfurt: Peter Lang, pp. 65-88.

Utsumi, A. (2000). "Verbal irony as implicit display of ironic environment: Distinguishing ironic utterances from nonirony", Journal of Pragmatics, 32 (12), pp. 1777-1806. https://doi.org/10.1016/ S0378-2166(99)00116-2

Wilson, D. (2006). “The pragmatics of verbal irony: Echo or pretence?", Lingua, 116 (10), pp. 17221743. https://doi.org/10.1016/j.lingua.2006.05.001 